\title{
Prevalence of Isolated "Pre-Malignant" Lesions on Prostate Biopsy in a Racially Diverse Community Screened Cohort
}

\author{
Michael A. Liss ${ }^{1}$, Donna Ankerst ${ }^{1,2}$, David Zapata1 ${ }^{1}$, Javier Hernandez ${ }^{1}$, Robin J. Leach ${ }^{1,3}$, \\ Ian M. Thompson ${ }^{1,4}$ \\ ${ }^{1}$ Department of Urology, University of Texas Health Science Center San Antonio, San Antonio, USA \\ ${ }^{2}$ Department of Epidemiology and Biostatistics, University of Texas Health Science Center San Antonio, San \\ Antonio, USA \\ ${ }^{3}$ Department of Cellular and Structural Biology, University of Texas Health Science Center San Antonio, San \\ Antonio, USA \\ ${ }^{4}$ Cancer Therapy and Research Center, University of Texas Health Science Center San Antonio, San Antonio, \\ USA \\ Email: *liss@uthscsa.edu
}

Received 30 October 2015; accepted 14 December 2015; published 17 December 2015

Copyright (C) 2015 by authors and Scientific Research Publishing Inc.

This work is licensed under the Creative Commons Attribution International License (CC BY).

http://creativecommons.org/licenses/by/4.0/

(c) () Op Den Access

\section{Abstract}

Objective: We investigated rates of prostate cancer (PCa), high-grade prostatic intraepithelial neoplaisa (HGPIN) and atypical small acinar proliferation (ASAP) in a multiethnic cohort. Methods: We evaluated prostate biopsy outcomes in men enrolled in the San Antonio Center of Biomarkers of Risk for prostate cancer (SABOR) prospective, observational study. PCa-free men underwent annual PSA testing over nearly 14 years with biopsies based on community standards. We investigated biopsy outcomes with a special interest in rates of cancer, HGPIN, and ASAP. Results: We identified 975 prostate biopsies in 801 subjects from 3/1/2001 to 1/9/2014. PCa, HGPIN, or ASAP was encountered in $28.8 \%$ (281/975), $10.1 \%(98 / 975)$, and $5.2 \%(51 / 975)$ of prostate biopsy specimens, respectively. The most significant risk factor for a PCa diagnosis was African American race (OR 5.0, 95\% CI: 2.2 - 11.4, $p<0.001$ ). HGPIN and ASAP occurred more commonly in association with PCa (both $p<0.001)$. We identified $57 \%(24 / 42)$ of men diagnosed with a "pre-malignant" lesion on prostate biopsy and had a subsequent biopsy. Of those only $8 \%(2 / 24)$ were diagnosed with prostate cancer (both Gleason $3+3$ ) within 1 year of the initial biopsy. Conclusion: We note a 5-fold increased risk of PCa for African American men. The incidence of HGPIN and ASAP are consistent with previously reported incidence. If diagnosed in isolation, repeat biopsy within one year could be delayed or eliminated as it may not change prostate cancer outcomes.

\footnotetext{
${ }^{*}$ Corresponding author.
} 


\section{Keywords}

\section{Active Surveillance, Ethnicity, Prostate Biopsy, Prostate Cancer, Race}

\section{Introduction}

Prostate cancer (PCa) is the most common solid organ malignancy in men with over 230,000 new cases expected in 2015 in the United States alone [1]. Transrectal ultrasound-guided systematic prostate biopsy is the most common means of diagnosing PCa with over 1 million performed annually in the United States [2] [3]. The proportion of men diagnosed with prostate cancer by prostate biopsy ranges between $27 \%-40 \%$, though it is affected by a number of variables [4] [5].

Race and ethnicity are directly related to prostate cancer risk and outcomes following treatment; however, some pathologic findings are also concerning for future risk of prostate cancer [6] [7]. The prevalence of two putatively premalignant lesions, high-grade prostatic intraepithelial neoplaisa (HGPIN) and atypical small acinar proliferation (ASAP), among different racial and ethnic groups has not been well characterized, especially in U.S. population-based studies [8]. Most studies to date have focused on the disproportionate risk of prostate cancer among African Americans and limited data are available on the rates of premalignant lesions in Hispanics, the largest and fastest-growing minority in the United States [9].

Hispanic men have similar rates of diagnosis and death from prostate cancer as non-Hispanic Whites, but are poorly-represented in clinical trials [10] [11]. The San Antonio Center of Biomarkers Of Risk for prostate cancer (SABOR) is a community based biomarker discovery and validation study that has followed a prospectivelyenrolled cohort with minority race/ethnicity oversampling since 2001; long-term follow-up is available in over 3000 men. Taking advantage of this cohort, we examined rates of prostate cancer, HGPIN, and ASAP to better understand how these rates correlated with overall rates of PCa and PCa aggressiveness in these populations.

\section{Methods}

\subsection{Study Population}

The San Antonio Center of Biomarkers Of Risk for prostate cancer (SABOR) is an Institutional Review Board approved Clinical Validation Center of the Early Detection Research Network of the National Cancer Institute. Since 2001, SABOR has recruited 3651 prostate cancer-free men at six sites into a longitudinal study. Data from prostate biopsies prompted by the study or performed prior to study enrollment are collected. Central pathology review was not performed. All biopsy reports were reviewed for presence of ASAP or HGPIN.

\subsection{Statistical Analysis}

The primary outcome of this study was the specific finding at prostate biopsy that included: PCa, ASAP, or HGPIN. Each prostate biopsy was treated as an independent event; however, a sub-analysis of only those men with a single prostate biopsy was performed due to different pre-test probabilities for cancer, depending on number of prior biopsies. Demographic data were analyzed using the un-paired two-tailed Student's t-test for continuous variables and the Pearson chi-squared test or Fisher's Exact test for binary variables. The difference in pathologic findings across the three racial/ethnic groups was examined using an analysis of variance (ANOVA) test. As the primary outcomes were binary, logistic regression was used in multivariable analysis. Variable selection was obtained by backward elimination using likelihood ratios. Statistical analysis was performed using SPSS v.21 (IBM, Chicago, IL, USA).

\section{Results}

\subsection{Subjects and Overall Pathology Findings}

We identified 975 prostate biopsies in 801 SABOR cohort subjects with complete pathology from men enrolled between 3/1/2001 to 1/9/2014; of these, repeat prostate biopsies accounted for $17.8 \%$ (174/975). The racial/ 
ethnic distribution of the biopsies was 60.0\% (580/975) Non-Hispanic White, 28.0\% (272/975) Hispanic White, and 12.6\% (123/975) African American. Study subject demographics are displayed in Table 1. Notable differences among racial/ethnic groups included that Hispanics had higher BMI $(\mathrm{p}<0.001)$ and African American men were younger $(\mathrm{p}<0.001)$. The number of biopsy cores obtained was not different among racial/ethnic groups with a median of 12 cores (ANOVA $\mathrm{p}=0.600$ ). Prostate cancer, HGPIN, or ASAP and were noted in 28.8\% (281/975), 10.1\% (98/975), and 5.2\% (51/975) of prostate biopsy specimens, respectively. Figure 1 (initial) and Figure 2 (repeat) display prostate biopsy pathology results by race and ethnicity.

\subsection{Prostate Cancer}

Of the 975 prostate biopsies, PCa was found in 29.1\% (233/801) of initial and 27.6\% (48/126) of repeat biopsies. Low-grade (Gleason 6 or less) cancer was the most common cancer diagnosis in all groups with non-Hispanic Caucasian, Hispanic Caucasians, and African Americans having similar rates: 61.5\%, 61.4\%, and 62.3\%, respectively $(\mathrm{p}=0.817)$. Variables predicting cancer presence at biopsy included African American race $(\mathrm{p}=$ $0.001)$, age ( $p=0.002)$, presence of HGPIN $(p \leq 0.001)$, and presence of ASAP $(p=0.027)$. Controlling for serum prostate specific antigen (PSA) levels, BMI, and prostate volume in multivariable logistic regression, the most significant factor for a prostate cancer diagnosis was African American race with an odds ratio of 5.0 (95\% CI: 2.2 - 11.4, $\mathrm{p}<0.001$ ). No difference was found in risk of prostate cancer between non-Hispanic Caucasian and Hispanic Caucasian men $(\mathrm{p}=0.874)$.

\subsection{High Grade PIN}

HGPIN was found in 9\% (72/801) of the initial biopsy specimens and in 14.9\% (26/174) of repeat biopsies (Fisher's Exact, p = 0.025). HPIN alone was diagnosed in 3.4\% (15/473) of initial biopsies and 5\% (5/100) of repeat biopsies. HGPIN was more likely to be diagnosed when prostate cancer was detected concurrently $(19.6 \%, 55 / 281)$ as compared to when no cancer was found on biopsy $(6.2 \%, 43 / 651)(p<0.001)$. There was no significant difference in the incidence of HGPIN among racial or ethnic groups: Non-Hispanic Caucasian $10.3 \%$ (60/580), Hispanic Caucasians 10.3\% (28/272) African American 8.1\% (19/123) (p = 0.750). In univariable analysis, larger prostate volume $(p=0.049)$, age $(p=0.004)$, and presence of prostate cancer $(p<0.001)$ were significant variables for a higher risk of HGPIN.

Table 1. Demographics ( $=975$ biopsy results).

\begin{tabular}{|c|c|c|c|c|}
\hline Demographic & $\begin{array}{c}\text { White } \\
\text { N (\%) or median } \\
\text { (IQR) } \\
\text { N }=580\end{array}$ & $\begin{array}{c}\text { Hispanic } \\
\text { N (\%) or median } \\
\text { (IQR) } \\
\text { N }=272\end{array}$ & $\begin{array}{l}\text { African American } \\
\text { N (\%) or median } \\
\text { (IQR) } \\
\mathbf{N}=123\end{array}$ & p value \\
\hline Age & $65(60-70)$ & $64(58-68)$ & $62(56-67)$ & $<0.001$ \\
\hline Body mass index & $27(25-30)$ & $29(26-31.5)$ & $29(27-32)$ & $<0.001$ \\
\hline Prostate specific antigen (ng/mL) & $3.2(1.7-4.8)$ & $3.5(1.9-5.2)$ & $3.1(1.8-4.7)$ & 0.916 \\
\hline Number of biopsy cores & $12(11-12)$ & $12(10-12)$ & $12(11-12)$ & 0.600 \\
\hline Prostate volume $\left(\mathrm{cc}^{3}\right)$ & $27(36-54)$ & $34(24-47)$ & $38(25-51)$ & 0.614 \\
\hline Prostate cancer & $169(29.1)$ & $70(25.7)$ & $42(34.1)$ & 0.224 \\
\hline Gleason 6 & 104 (61.5) & 43 (61.4) & $28(66.7)$ & \multirow[b]{2}{*}{0.817} \\
\hline Gleason 7+ & $65(38.5)$ & $27(38.6)$ & 14 (33.3) & \\
\hline High grade PIN & $60(11.6)$ & $28(11.5)$ & $10(9.7)$ & 0.856 \\
\hline Atypical small acinar proliferation & 36 (6.9) & $13(5.3)$ & $2(3.9)$ & $0.131^{*}$ \\
\hline
\end{tabular}

\footnotetext{
${ }^{*}$ Linear association $\mathrm{p}=0.05$.
} 
Pathology of First Biopsy in SABOR

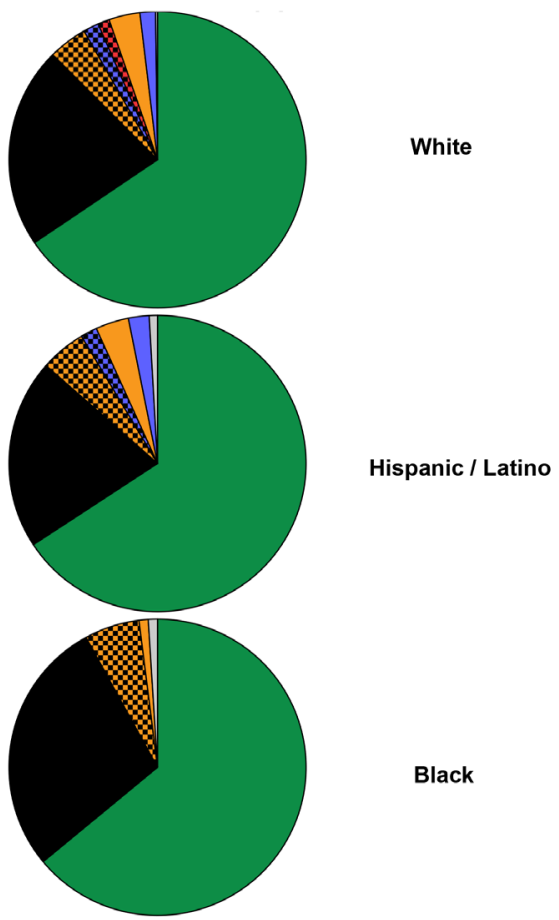

\begin{tabular}{|c|c|c|}
\hline $\mathrm{N}$ & $(\%)$ & Pathology \\
\hline 520 & 64.9 & Benign \\
\hline 178 & 22.2 & Cancer \\
\hline 37 & 4.6 & BCancer + HGPIN \\
\hline 12 & 1.5 & BCancer + ASAP \\
\hline 6 & 0.7 & BCancer + HGPIN + ASAP \\
\hline 25 & 3.1 & $\square$ HGPIN \\
\hline 13 & 1.6 & $\square$ ASAP \\
\hline 4 & 0.5 & $\square \mathrm{HGPIN}+\mathrm{ASAP}$ \\
\hline 5 & 0.6 & Missing/Excluded \\
\hline
\end{tabular}

Figure 1. Pathology of first biopsy documented in SABOR database. A pie chart graphically represents the pathologic outcomes of the first documented prostate biopsy among SABOR participants. Eight colors represent the various combinations of benign, malignant, ASAP, and HGPIN.
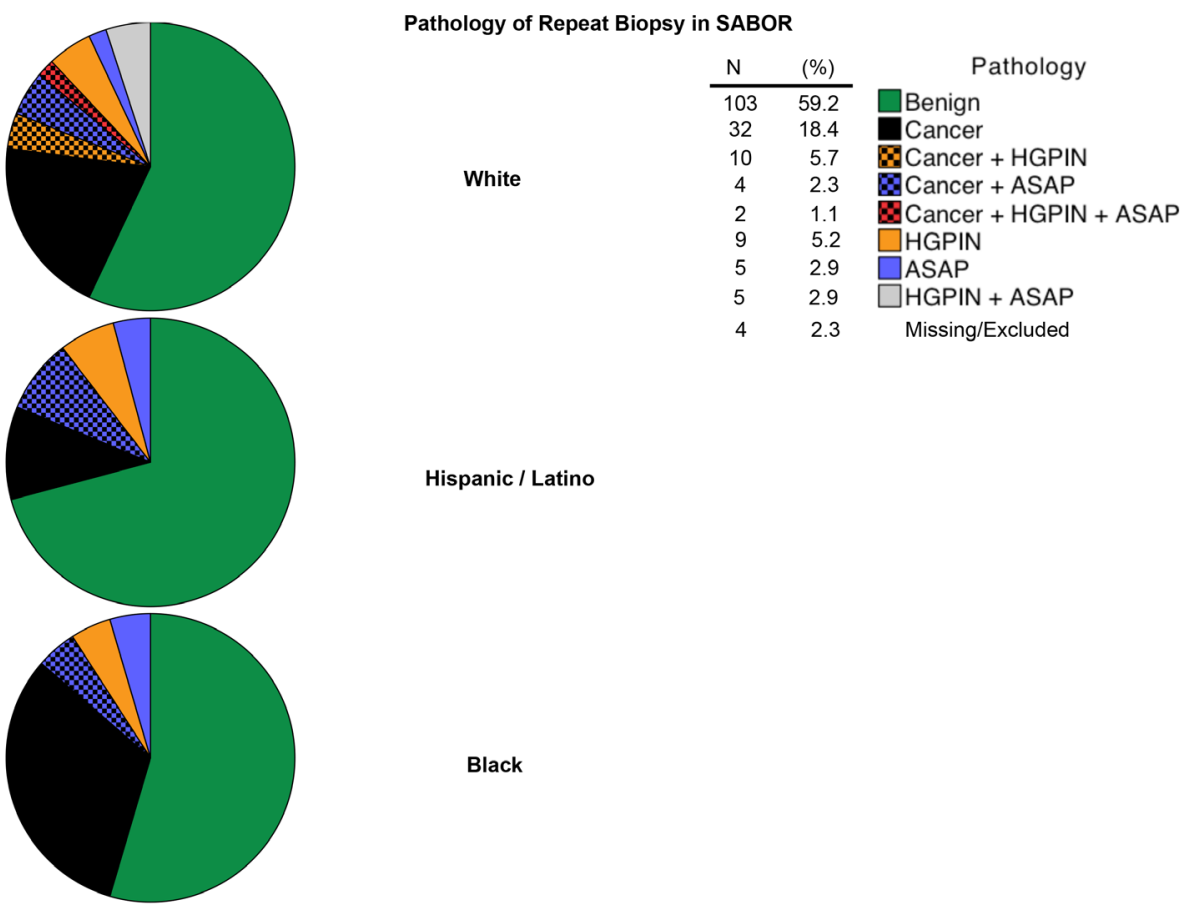

Hispanic / Latino

Black

Figure 2. Pathology of repeat biopsy documented in SABOR database. A pie chart graphically represents the pathologic outcomes of the repeated prostate biopsies documented prostate biopsy among SABOR participants. Eight colors represent the various combinations of benign, malignant, ASAP, and HGPIN. 


\subsection{Atypical Small Acinar Proliferation}

ASAP was diagnosed in 4.4\% (35/801) of biopsies and 9.2\% of repeat prostate biopsies 9.2\% (16/174) (Fisher's Exact $\mathrm{p}=0.010)$. ASAP was more likely to be diagnosed with concurrent PCa [8.5\% (24/281)] than in isolation [3.9\% $(27 / 694)](\mathrm{p}=0.003)$. We found no statistical difference in the incidence of ASAP on prostate biopsy when examined by race/ethnicity: non-Hispanic Caucasians 6.2\% (36/580), Hispanic Caucasians 4.8\% (13/272), and African American 1.6\% (2/121) $(\mathrm{p}=0.108$, $\mathrm{p}$ for trend 0.040). Of interest, ASAP did appear less-common among African Americans. Variables positively associated with ASAP included age $(p=0.001)$ and prostate cancer $(p=0.027)$ with a trend related to higher prostate volume $(p=0.060)$.

\subsection{Repeat Biopsy after ASAP or HGPIN}

We identified only 57\% (24/42) of men diagnosed with a "pre-malignant" lesion on prostate biopsy had a subsequent biopsy. The "pre-malignant" lesions included: HGPIN $(n=11)$, ASAP $(n=9)$, or HGPIN + ASAP $(n=$ 4) (Table 2). Of men diagnosed with ASAP or ASAP/HGPIN alone on a biopsy without a previous diagnosis of prostate cancer, 8/13 had a prostate biopsy within 1 year; however, only 2 were diagnosed with PCa on the repeat biopsy and both cancers were low-grade (Gleason $3+3$ ). Eventually, 7/11 men were diagnosed with PCa at

Table 2. Patients with isolated HGPIN or ASAP that underwent repeat biopsy.

\begin{tabular}{|c|c|c|c|c|c|c|}
\hline Race & $\begin{array}{c}\text { Initial } \\
\text { pathology }\end{array}$ & $\begin{array}{l}\text { Repeat biopsy } \\
\text { within } 1 \text { year }\end{array}$ & Cancer & Gleason & $\begin{array}{c}\text { Years to } \\
\text { cancer diagnosis }\end{array}$ & $\begin{array}{c}\text { Number } \\
\text { of biopsies }\end{array}$ \\
\hline Mexican & ASAP & $\mathrm{x}$ & Cancer & $3+4$ & 8.06 & 2 \\
\hline White & ASAP & $\mathrm{x}$ & Cancer & $3+3$ & 10 & 2 \\
\hline White & ASAP & Cancer & Cancer & $3+3$ & 1.12 & 2 \\
\hline White & ASAP & Negative & Cancer & $3+3$ & 3.2 & 4 \\
\hline White & ASAP & Negative & Cancer & $3+3$ & 6.01 & 3 \\
\hline Mexican & ASAP & Negative & & & & 2 \\
\hline White & ASAP & Cancer & Cancer & $3+3$ & 0.73 & 4 \\
\hline Mexican & ASAP & Negative & & & & 5 \\
\hline Mexican & ASAP & Negative & & & & 2 \\
\hline White & ASAP/HGPIN & $\mathrm{x}$ & Cancer & $3+3$ & 5.46 & 2 \\
\hline White & ASAP/HGPIN & $\mathrm{x}$ & & & & 2 \\
\hline Mexican & ASAP/HGPIN & $\mathrm{x}$ & & & & 2 \\
\hline White & ASAP/HGPIN & Negative & & & & 4 \\
\hline Black & HGPIN & $\mathrm{x}$ & Cancer & $3+3$ & 2.26 & 3 \\
\hline White & HGPIN & $\mathrm{x}$ & & & & 3 \\
\hline White & HGPIN & Negative & & & & 4 \\
\hline Mexican & HGPIN & $\mathrm{x}$ & & & & 3 \\
\hline Puerto Rican & HGPIN & $\mathrm{x}$ & & & & 2 \\
\hline White & HGPIN & $\mathrm{x}$ & & & & 2 \\
\hline Mexican & HGPIN & $\mathrm{x}$ & & & & 2 \\
\hline White & HGPIN & Negative & Cancer & $4+5$ & 1.84 & 4 \\
\hline White & HGPIN & $\mathrm{x}$ & & & & 4 \\
\hline Black & HGPIN & Negative & Cancer & $3+3$ & 1.72 & 3 \\
\hline White & HGPIN & ASAP & Cancer & & 3.8 & 4 \\
\hline
\end{tabular}


a median of 5.5 years (range 0.7 - 10.0 years). For men with HGPIN alone, 5/11 had another biopsy within one year with no cancer diagnosis. Eventually, 2/11 men were diagnosed with prostate cancer at a median of 2 years (1.7 - 3.8 years) from the biopsy with HGPIN (Table 2).

\section{Discussion}

The overall diagnostic rate for premalignant lesions not associated with overt prostate cancer remained low, though clinically significant $(4.3 \%, 42 / 975)$. Extrapolating this data to an estimated 1 million biopsies are performed in the United States each year, a "pre-malignant" diagnosis could affect nearly 43,000 men per year. Our data provide further support to concerns that HGPIN or ASAP have either a small or no impact on intermediate prostate cancer outcomes. Others have suggested that rates of HGPIN, atypical, non-definitive diagnoses, and low-grade cancer could correlate with diagnostic habits [12]. Therefore, our data suggest that there may be little benefit from standardized repeat biopsy recommendations based on the diagnosis of ASAP or HGPIN in isolation on prostate biopsy. In our study, most of these patients were not diagnosed with cancer on a biopsy performed within one year of the diagnosis of HGPIN or ASAP, suggesting these biopsies could likely be delayed or eliminated. Perhaps more importantly, if PCa was diagnosed, most were low grade, tumors that benefit little from detection or treatment [13]. Our findings are in contrast to previously recommendations for extended biopsies or even prostatectomy for these pathologic entities due to reportedly higher rate of associated cancer, albeit largely low-grade [14] [15]. Given the significant attention given to over-detection (and thus overtreatment as well) of prostate cancer, our data provide support to a more conservative approach to HGPIN and ASAP.

The SABOR cohort is a unique resource for examining prostate cancer risk and characteristics in an aging, multi-ethnic, multi-racial population, one that better mirrors U.S. outcomes than studies from Urology practices that may be affected by referral patterns and often have more homogeneous racial/ethnic demographics. SABOR has facilitated examination of many differences among racial and ethnic groups [12] [16] [17]. The observation of the dramatically higher risk of prostate cancer among African American men is consistent with national data [1]. Of interest is the slightly-lower detection rate of prostate cancer (25.7\%) among other racial/ethnic groups [3] [4].

We sought to explore pathologic outcomes in a more detailed fashion. In this analysis, the incidence of HGPIN and ASAP did not vary substantially among racial and ethnic groups. Curiously, while African American men had higher rates of cancer, although not significant, a measurably lower rate of ASAP was found in these men in SABOR. We did confirm our previous observation from the Prostate Cancer Prevention Trial that age has a significant effect on the risk of prostate cancer [18].

At the prostate cancer level, these data confirm the importance of focusing early detection efforts on African American men. We have previously demonstrated the importance of the PCPT Risk Calculator to incorporate this variable in decision-making for prostate biopsies due to the significantly greater risk of cancer and of high-grade prostate cancer in this racial group [19].

There are several limitations to this study including the lack of central pathologic review and a relatively small number of African American men. Regarding the former, our data become more generalizable as a broad range of nuance of pathologic interpretation was operational. Regarding the later, a major study strength is the broad range of races/ethnicities included, especially Hispanic men who are underrepresented in these studies and constitute the fastest growing ethnic group in the U.S. A major strength of this study was the prolonged follow-up.

\section{Conclusion}

In this population-based cohort study with prolonged follow-up, we found a 5-fold increased risk of prostate cancer in African American men. HGPIN and ASAP were common pathologic findings but were unassociated with risk of prostate cancer.

\section{Acknowledgements}

We would like to acknowledge our SABOR patients who have enrolled and the research staff at UTHSCSA for their tireless efforts. Dr. Michael Liss is funded by DOD PC141535. Ian Thompson is the principal investigator of SABOR funded by U01 CA086402 and P30 CA054174 mechanisms of the National Institutes of Health. 


\section{Conflict of Interest}

None.

\section{References}

[1] Siegel, R.L., Miller, K.D. and Jemal, A. (2015) Cancer Statistics, 2015. CA: A Cancer Journal for Clinicians, 65, 5-29. http://dx.doi.org/10.3322/caac.21254

[2] Seltzer, M.A., Shinohara, K., Bhargava, V., Carroll, P.R. and Presti Jr., J.C. (1996) Comparison of Systematic Sextant and Lesion Directed Biopsies in Prostate Cancer Detection. Urologic Oncology, 2, 136-138. http://dx.doi.org/10.1016/S1078-1439(96)00092-0

[3] Welch, H.G., Fisher, E.S., Gottlieb, D.J. and Barry, M.J. (2007) Detection of Prostate Cancer via Biopsy in the Medicare-SEER Population during the PSA Era. Journal of the National Cancer Institute, 99, 1395-1400. http://dx.doi.org/10.1093/jnci/djm119

[4] Kapoor, D.A., Bostwick, D.G., Mendrinos, S.E., Anderson, A.E. and Olsson, C.A. (2013) Utilization Trends and Positive Biopsy Rates for Prostate Biopsies in the United States: 2005 to 2011. Reviews in Urology, 15, 137-144.

[5] Patel, A.R. and Jones, J.S. (2009) Optimal Biopsy Strategies for the Diagnosis and Staging of Prostate Cancer. Current Opinion in Uurology, 19, 232-237. http://dx.doi.org/10.1097/MOU.0b013e328329a33e

[6] Fowler Jr., J.E., Bigler, S.A. and Farabaugh, P.B. (2002) Prospective Study of Cancer Detection in Black and White Men with Normal Digital Rectal Examination but Prostate Specific Antigen Equal or Greater than 4.0 ng/mL. Cancer, 94, 1661-1667. http://dx.doi.org/10.1002/cncr.10446

[7] Mullins, C.D., Onukwugha, E., Bikov, K., Seal, B. and Hussain, A. (2010) Health Disparities in Staging of SEERMedicare Prostate Cancer Patients in the United States. Urology, 76, 566-572. http://dx.doi.org/10.1016/j.urology.2009.10.061

[8] Epstein, J.I. and Herawi, M. (2006) Prostate Needle Biopsies Containing Prostatic Intraepithelial Neoplasia or Atypical Foci Suspicious for Carcinoma: Implications for Patient Care. The Journal of Urology, 175, 820-834. http://dx.doi.org/10.1016/S0022-5347(05)00337-X

[9] Gomez, P., Manoharan, M., Sved, P., Kim, S.S. and Soloway, M.S. (2004) Radical Retropubic Prostatectomy in Hispanic Patients. Cancer, 100, 1628-1632. http://dx.doi.org/10.1002/cncr.20127

[10] Siege, R., Ma, J., Zou, Z. and Jemal, A. (2014) Cancer Statistics, 2014. CA: A Cancer Journal for Clinicians, 64, 9-29. http://dx.doi.org/10.3322/caac.21208

[11] Pinsky, P.F., Ford, M., Gamito, E., et al. (2008) Enrollment of Racial and Ethnic Minorities in the Prostate, Lung, Colorectal and Ovarian Cancer Screening Trial. Journal of the National Medical Association, 100, 291-298.

[12] Weinstein, M.H., Greenspan, D.L. and Epstein, J.I. (1998) Diagnoses Rendered on Prostate Needle Biopsy in Community Hospitals. The Prostate, 35, 50-55. http://dx.doi.org/10.1002/(SICI)1097-0045(19980401)35:1<50::AID-PROS7>3.0.CO;2-L

[13] Thompson Jr., I.M., Leach, R.J. and Ankerst, D.P. (2014) Focusing PSA Testing on Detection of High-Risk Prostate Cancers by Incorporating Patient Preferences into Decision Making. JAMA, 312, 995-996. http://dx.doi.org/10.1001/jama.2014.9680

[14] Ploussard, G., Plennevaux, G., Allory, Y., et al. (2009) High-Grade Prostatic Intraepithelial Neoplasia and Atypical Small Acinar Proliferation on Initial 21-Core Extended Biopsy Scheme: Incidence and Implications for Patient Care and Surveillance. World Journal of Urology, 27, 587-592. http://dx.doi.org/10.1007/s00345-009-0413-1

[15] Brausi, M., Castagnetti, G., Dotti, A., De Luca, G., Olmi, R. and Cesinaro, A.M. (2004) Immediate Radical Prostatectomy in Patients with Atypical Small Acinar Proliferation. Over Treatment? The Journal of Urology, 172, 906-908. http://dx.doi.org/10.1097/01.ju.0000134622.54235.93

[16] Feneley, M.R., Green, J.S., Young, M.P., et al. (1997) Prevalence of Prostatic Intra-Epithelial Neoplasia (PIN) in Biopsies from Hospital Practice and Pilot Screening: Clinical Implications. Prostate Cancer and Prostatic Diseases, 1, 79-83. http://dx.doi.org/10.1038/sj.pcan.4500210

[17] Gupta, C., Ren, J.Z. and Wojno, K.J. (2004) Individual Submission and Embedding of Prostate Biopsies Decreases Rates of Equivocal Pathology Reports. Urology, 63, 83-86. http://dx.doi.org/10.1016/j.urology.2003.09.016

[18] Thompson, I.M., Ankerst, D.P., Chi, C., et al. (2006) Assessing Prostate Cancer Risk: Results from the Prostate Cancer Prevention Trial. Journal of the National Cancer Institute, 98, 529-534. http://dx.doi.org/10.1093/jnci/djj131

[19] Ankerst, D.P., Hoefler, J., Bock, S., et al. (2014) Prostate Cancer Prevention Trial Risk Calculator 2.0 for the Prediction of Low- vs High-Grade Prostate Cancer. Urology, 83, 1362-1367. http://dx.doi.org/10.1016/j.urology.2014.02.035 